\title{
The Radiation of Light. ${ }^{1}$
}

\section{By Prof. H. A. Lorentz, For. Mem. R.S., University of Leyden.}

$\mathrm{O}^{\mathrm{N}}$ $\mathrm{NE}$ of the lessons which the history of science teaches us is surely this, that we must not too soon be satisfied with what we have achieved. The way of scientific progress is not a straight one which we can steadfastly pursue. We are continually seeking our course, now trying one path and then another, many times groping in the dark, and sometimes even retracing our steps. So it may happen that ideas which we thought could be abandoned once for all, have again to be taken up and come to new life.

These remarks are well illustrated by the way in which at different times physicists have represented to themselves the way in which light is produced and radiated. The two contending views, the emission theory or the corpuscular theory of light, developed by Newton, and the undulatory theory proposed by Huygens, perfected afterwards by Young and Fresnel, and newly shaped as the electromagnetic theory of light by Clerk Maxwell, are well known. I should now like to point out how these two theories, however widely different their principles may be, were interwoven in Newton's mind, and how it is well possible that they will be interwoven again in the physics of the future.

When one reads Newton's "Optics," one cannot doubt that, when he speaks of a ray of light, he always thinks of a stream of small corpuscles emitted by a luminous body and moving along in straight lines, so long as they are not acted upon by some deflecting force. The phenomenon of diffraction, as we call it now, that had been discovered by Grimaldi and which Newton carefully examined experimentally, I mean the phenomenon that the shadow of a thin wire, for example, is wider than it would be in the case of undisturbed rectilinear propagation, was attributed by Newton to certain repulsive forces with which the wire acts upon the rays of light when they pass along its surface at a very small distance.

The reflection and the refraction of light were likewise considered as due to forces which act upon the corpuscles of a ray when they come near the surface of separation of two media, like air and glass or water. Newton expressly states that the corpuscles must not be conceived to be reflected by the individual molecules of the body on which they impinge. If we could see the molecules, we should find the surface to be very rough, and it would be clear that their individual actions can scarcely lead to a regular specular reflection. For this reason Newton supposes that the corpuscles of light are acted upon, not by the molecules separately, but by parts of the bodies containing a great number of them. This does not prevent us from supposing the actions in question to be appreciable at very small distances only. If they were sensible up to one tenthousandth of a millimetre, for example, and if you had a corpuscle situated at half that distance from a polished plate of glass, then, since the structure of the glass is very fine grained with respect to the distance in question, the corpuscle would be acted upon by an immense number of molecules, and the discontinuities would not make themselves felt, so that it would be as if we had a perfectly smooth surface.

1 Discourse delivered at the Royal Institution on Friday, June r, r923.

$$
\text { NO. } 2843 \text {, vOL. I I } 3 \text { ] }
$$

The forces of which I am speaking are comparable to those that were introduced much later by Laplace in the theory of capillarity. A corpuscle in the interior of a body will experience equal forces in all directions, so that there will be no resulting force and the particle continues its way with a constant velocity. It is only in a very thin layer extending on both sides of the surface of separation, that there will be a resulting force, due to the unequal attractions, or perhaps repulsions, which the two media exert on the corpuscle. For reasons of symmetry the resulting force is perpendicular to the surface of separation, and therefore, whether the corpuscle be reflected into the first medium or allowed to continue its way in the second, the component of the velocity in the direction of the surface will remain unchanged. From this you can easily deduce the law of reflection. As to that of refraction, a simple diagram (Fig. $x$ ) shows that, if the velocity along the surface $A B$ is the same before and

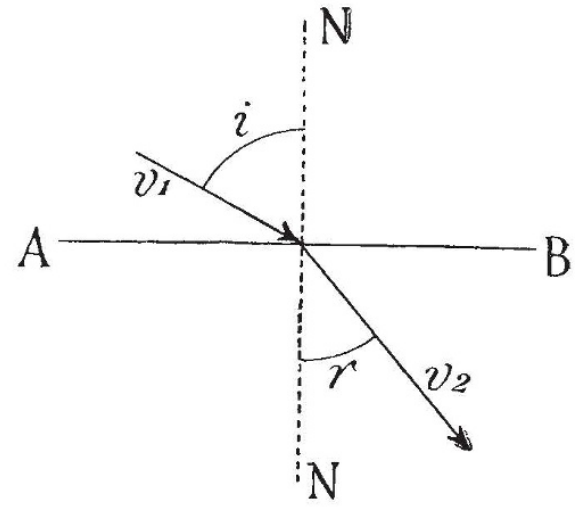

FIG. I.

after the refraction, the sines of the angles which the ray makes with the normal $\mathrm{NN}$, the angle of incidence $i$ and the angle of refraction $r$, will be inversely proportional to the velocities, say $v_{1}$ and $v_{2}$, with which the corpuscles move on the two sides of the surface; indeed, since the component of the velocity along the surface is $v_{1} \sin i$ on one side, and $v_{2} \sin r$ on the other, its constancy requires that

$$
\sin i: \sin r=v_{2}: v_{1} \text {. }
$$

We shall therefore have found the well-known law of refraction, namely, the constancy of the ratio between the two sines, if we suppose $v_{1}$ and $v_{2}$ to have definite values, whatever be the angle of incidence. Now, if the first medium is air, or rather the ether, we shall suppose $v_{1}$ to have a definite value, at least for rays of a particular colour, on account of the way in which light is emitted. Once taking for granted this constancy of $v_{1}$, we can assure ourselves of that of $v_{2}$ by taking into account that the passage of a corpuscle from the interior of the first medium to the interior of the second will be accompanied by a definite change in the value of the potential energy, this value being in each medium the same for all positions of the corpuscle and whatever be its motion. By the law of the conservation of energy the change in the kinetic energy 
of the corpuscle must be equal and opposite to that of the potential energy; thus, the difference $v_{1}^{2}-v_{2}^{2}$ must be the same in all cases, and the constancy of $v_{2}$ follows from that of $v_{1}$.

I shall dwell no longer upon the further development of the corpuscular theory, but I must now point out that Newton also had the notion of vibrations, and even of waves, that are propagated in a medium. $\mathrm{He}$ was led to this by his experiments on the colours of thin plates, which we now attribute to interference, and which he examined very carefully for the case of the thin layer of air contained between a plane surface of glass and a surface that is slightly convex. Here we have the phenomenon generally known as Newton's rings. Suppose the light to be homogeneous and the incidence to be normal. Then, both in the reflected and in the transmitted light, you will see a number of alternating bright and dark rings, the dark rings in the transmitted light having the same diameters as the bright ones in the reflected light. This means of course, what is quite natural, that, at a thickness of the layer for which a great part of the light is reflected, only a small part is transmitted, and conversely.

The experiment shows that, of all the rays that enter at the front surface of the layer, some will be reflected at the back surface and others not, and that this depends on the thickness of the layer, the distance between the two surfaces. Further, that there is a certain periodicity in this. Now, since two rays, one of which is reflected at the back surface and the other not, must obviously be more or less different, Newton supposes that, after having passed the first surface, a ray or a corpuscle is in alternating states, fits, as he calls them, of easy reflection and easy transmission. But I think I had better give this in his own words :-

What kind of action or disposition this is ; whether it consists in a circulating or a vibrating motion of the Ray, or of the Medium, or something else, I do not here enquire. Those that are averse from assenting to any new Discoveries, but such as they can explain by an Hypothesis, may for the present suppose, that as Stones by falling upon Water put the Water into an undulating Motion, and all Bodies by percussion excite vibrations in the Air; so the Rays of Light, by impinging on any refracting or reflecting Surface, excite vibrations in the refracting or reflecting Medium or Substance, and by exciting them agitate the solid parts, of the refracting or reflecting Body, and by agitating them cause the Body to grow warm or hot; that the vibrations thus excited are propagated in the refracting or reflecting Medium or Substance, much after the manner that vibrations are propagated in the Air for causing Sound, and move faster than the Rays so as to overtake them; and that when any Ray is in that part of the vibration which conspires with its Motion, it easily breaks through a refracting Surface, but when it is in the contrary part of the vibration which impedes its Motion, it is easily reflected; and, by consequence, that every Ray is successively disposed to be easily reflected, or easily transmitted, by every vibration which overtakes it. But whether this Hypothesis be true or false I do not here consider. I content my self with the bare Discovery, that the Rays of Light are by some cause or other alternately disposed to be reflected or refracted for many vicissitudes.

Let me add that, in order to account for the fact that light falling on a surface of glass is partially reflected and partially transmitted, Newton assumed that fits of easy transmission and easy reflection exist already in the incident rays before they reach the surface. He supposed these fits to have been impressed on the rays already in the act of emission itself ; in fact he went so far as to imagine something like vibrations to go on in the source of light. Query VIII near the end of the book begins with the words :-

Do not all fix'd Bodies, when heated beyond a certain degree, emit Light and shine; and is not this Emission perform'd by the vibrating motions of their parts?

So there was much of vibratory or undulatory theory in Newton's ideas, though he seems never to have thought, as Huygens did, of the ray itself consisting in a propagation of waves. In Query XVII he again compares the ray of light falling on the surface of some substance to a stone thrown into stagnant water.

As is well known, the conclusion that the sines of the angles of incidence and refraction would be inversely proportional to the velocities of propagation became fatal to the corpuscular theory. The undulatory theory required that the two sines be directly proportional to the velocities of propagation, and when, about a century ago, the velocity of light in water could be measured, the result was in full agreement with the wave theory. I can briefly state the facts by saying that the index of refraction of water is about $4 / 3$. The velocity of light in water ought therefore to be $3 / 4$ of that in air according to the undulatory theory, and so it was found to be, but the corpuscular theory requires that it should be greater than the velocity in air, namely, $4 / 3$ times that velocity.

There now came a period during which the wave theory reigned supreme, until in these last ten or twenty years physicists have been led to ideas, not exactly the same as Newton's, but still more or less similar to the notions of the corpuscular theory.

The beginning of it was that, on the basis of the electromagnetic theory, a beam of light was recognised to possess a certain momentum, comparable to that of a moving ball. For the ball the momentum is given by the product $m v$ of the mass and the velocity, and when we attribute to the beam of light a certain momentum, say an amount $\mathrm{Q}$ of it, we simply mean to say that the beam has the same power of setting bodies in motion as a body would have, for which the product $m v$ has just that value $\mathrm{Q}$.

The existence of momentum in a beam of light is shown by the pressure of radiation that was predicted by Clerk Maxwell, and observed and measured first by Lebedew and afterwards by E. F. Nichols and Hull. Let us consider this question for the case of a beam of light falling normally on a perfectly reflecting mirror, and let us compare the explanation by the undulatory theory, and the explanation that could be given by a follower of Newton, if there were one in these times. For the sake of simplicity I shall suppose that we hold the mirror in position by applying to it a certain force. If we can calculate that force we shall also know the pressure on the mirror, to which it is equal.

In the experiments the pressure has been compared to the energy which, in the beam of light, falls on the mirror in unit time, the two quantities being

NO. 2843 , VOL. I I 3$]$ 
proportional to each other. Now, according to both theories, the momentum which falls on the mirror has its direction reversed, and the ratio in question will be equal to that of twice the momentum of the light that reaches the surface during a certain time, to the energy of that same light. In the corpuscular theory this would be the ratio of $2 m v$ to $\frac{1}{2} m v^{2}$, or $4 / v$; thus, when $c$ is the speed of light, $4 / c$. On the undulatory theory the ratio between the momentum and the energy of a beam is that of $\mathrm{I}$ to $c$, by which the result becomes 2/c. Thus, for a beam of a given intensity, the pressure would be different in the two theories, in the undulatory theory half only of what it would be on the other view, so that here again we have a crucial experiment. The measurements have clearly decided in favour of the wave theory.

Our neo-Newtonian would have to own himself defeated by this, if he had been taught classical mechanics only, and had never heard of the changes that have been brought about by the theory of relativity. If he has studied this latter theory there is an escape for him ; indeed, he can point out triumphantly that the values which relativity assigns to the momentum and the energy of any moving system are such that our last result $2 / c$ holds in ail cases. Only, this appeal to relativity would imply that the corpuscles become widely different from what they were originally thought to be. According to relativity dynamics, a thing moving with the speed of light and having a finite mass, however small it may be, would have an infinite momentum and an infinite energy. Therefore, since the pressure has a finite magnitude, a corpuscle must be something with no mass $m$ at all, but which, nevertheless, when moving with the velocity $c$, has a finite energy and momentum. By these assumptions the corpuscles become much like the so-called light quanta of modern theory, to which I should now like to refer.

The word "quanta" is used by physicists in two different senses. In some cases we mean by it no more than definite amounts of energy of radiation, the magnitude of which is proportional to the frequency $n$, or number of vibrations in unit of time, so that it can be represented by $h n$, where $h$ is a constant. In this form the idea originated with Planck, who used it in the problems of heat radiation, and after whom the constant $h$ is generally called. In Bohr's theory of spectral lines these minute amounts of energy play a fundamental and most important part; one of his assumptions being that light is not emitted in quantities of any magnitude, but in a greater or smaller number of full quanta that are radiated successively, one at a time.

It ought to be remarked that in this form the notion of quanta has nothing that is very startling or mysterious. If a tuning fork is made to vibrate by taps of a definite intensity, the fork being allowed to lose all its energy before it receives a new blow, we shall have emission of " sound quanta." We can imagine without difficulty that similarly in a source of light the energy is measured out in small but finite portions of a fixed magnitude.

However, this does not always suffice. There are phenomena from which, if we had to judge by them solely, we should certainly infer that the energy of a quantum not only has a definite amount, but also remains confined to a very small space. In this way one has been led to the idea of "concentrated quanta," which may well be said to be Newton's corpuscles in a modernised form.

The phenomena to which I alluded are those of photo-electricity. When light of a suitable frequency is let fall on a plate of a properly chosen substance, electrons are set free, and it has been found that the energy of each of these electrons is equal to the quantum for the light which we use. This can be easily understood if the quanta are confined to small spaces, so that the electron can catch at once a whole quantum, whereas, if a quantum is spread out over a considerable extent, it is very difficult to see how an electron is to get hold of it. So the phenomena of photo-electricity seem to speak in favour of some corpuscular theory.

Let us, in order to make this clearer, suppose that the sensitive plate is first placed at a small distance from the source of light, and is then removed to a distance a hundred times as great, so that the intensity of the light or the total energy that falls on the plate in a certain time becomes ro,000 times less. Observation shows that the number of electrons liberated from the plate also becomes ro,ooo times less, but that they are ejected with exactly the same velocity as before. This would be very natural if we could adopt some form of corpuscular theory, either the old or the modernised one. Then it would be clear that the number of corpuscles striking the plate has diminished in the ratio I mentioned, but that each individual corpuscle can do just what it did at the smaller distance, for the velocity has not been altered, and the corpuscle or the concentrated quantum has lost none of its properties.

On the contrary, when there are no concentrations, when, in spreading out, the energy becomes more and more dilute, we should expect that, at a certain distance, the light becomes too feeble ever to liberate an electron.

So it would seem that we really want concentrated quanta. But now, having recognised this, we have to face a very serious difficulty, a difficulty that hangs as a heavy cloud over this part of physics. Indeed, the existence of narrowly limited disturbances of equilibrium is absolutely irreconcilable with the principles of the undulatory theory as they are embodied, for example, in Maxwell's equations of the electromagnetic field. According to these equations, a disturbance of the state of the ether can never remain confined within a space of constant magnitude; around each point that is reached by the disturbance there is a propagation in all directions, and so there is always the tendency to a lateral expansion that becomes manifest in the phenomena of diffraction. It is true that, when our openings are wide in comparison with the wave-length, we may have beams of light that are rather sharply defined over a certain length, but if we go far enough along the beam we shall ultimately notice an unlimited expansion. When, for example, parallel rays are made to pass through an opening of one centimetre in diameter, we observe an illuminated circle of the same magnitude with a rather sharp border on a screen at a distance of some metres, but if the screen is removed to a distance of roo kilometres, there will

$$
\text { NO. } 2843 \text {, VOL. I I } 3]
$$


be a badly defined patch of light extending over something like half a metre. Or, again, take the case of a disturbance initially confined within a spherical space a centimetre in diameter. At some later instant it will be found in a shell of this thickness, bounded by two concentric spheres which both expand with the speed of light. By properly choosing the distribution of the disturbance in the initial sphere, you have it in your power to produce different distributions in the expanding shell, but you can never prevent the disturbance from occupying ultimately a very considerable part of the spherical wave.

One might object that these are mostly theoretical inferences and that we must never swear by a theory, not even by Maxwell's. Let me therefore conclude by pointing out that, so far as we can see now, the hypothesis of concentrated quanta is directly in contradiction with observed facts, namely, with what is seen in the phenomena of interference.

It is known that bright and dark interference fringes can never be produced by means of two different, mutually independent, sources of light; we explain this by the want of all coherence between the vibrations in one source and those in the other. Now, the elementary acts of emission, in each of which a quantum is radiated, must certainly be incoherent; they may take place in different atoms, and there is not the least reason why there should be any connexion between what goes on in one atom and in another. Hence, when we observe an interference phenomenon, one quantum taken by itself must be able to produce it, and this will enable us to draw some conclusions concerning the extension of a quantum in different directions.

In certain experiments made with highly homogeneous light, interference fringes have been observed, produced by rays the paths of which differed by more than a million of wave-lengths. This means that there was a regular succession of more than a million of waves, and, since all these waves must be contained in one quantum, the length of a quantum in the direction of propagation must have been more than, say, $50 \mathrm{~cm}$. That the lateral dimensions must be no less considerable is shown by the influence which the aperture of an optical instrument has on the quality of the images, and consequently on the resolving power. Let $\mathrm{L}$ (Fig. 2) be the objective glass of a telescope, a " perfect" lens from the point of view of geometrical optics, so that, if there were no diffraction, the rays $\mathrm{R}$ coming from a distant star would converge exactly towards the focus $F$. In reality this can never be; on the plane $\mathrm{V}$ there will be an illuminated spot of a certain extent, and if we want to have this spot small, so that there is a sharply defined image of the star, we must use a large lens. As a matter of fact this is one of the advantages of the great modern telescopes.

Let us conceive the opening of the telescope to be divided into two parts, say of equal areas, a central circle $L_{1}$ and a ring $L_{2}$ around it. If, by means of a screen with a circular opening, we reduce the aperture to the part $\mathrm{L}_{1}$, the image of the star becomes less sharp ; a point $\mathrm{P}$ at a small distance from $\mathrm{F}$, which remains dark when the full aperture is used, may now be illuminated, the light disappearing again when the screen before the lens is removed. The explanation is, of course, that the vibrations which $\mathrm{P}$ receives from $\mathrm{L}_{1}$ are counteracted by opposite ones coming from the part $\mathrm{L}_{2}$. So, the fact that a large opening actually has the effect on the quality of the image which we expected from it, shows that there is interference between vibrations reaching the lens at different points of its surface. According to what we saw, these vibrations must belong to one and the same quantum, and we may therefore conclude that the lateral extension of a quantum is comparable to the size of the objective.

The remarkable experiments by which Prof. Michelson has been able to measure the diameter of some stars allow us to push the argument still further. In his apparatus a beam carrying four mirrors, $1,2,3,4$, was placed in front of the opening of the telescope, the mirrors 2 and 3 occupying places within the opening, whereas I and 4 were outside the opening, on opposite sides of it. The mirrors were adjusted in such a way that two beams of rays coming from the star entered

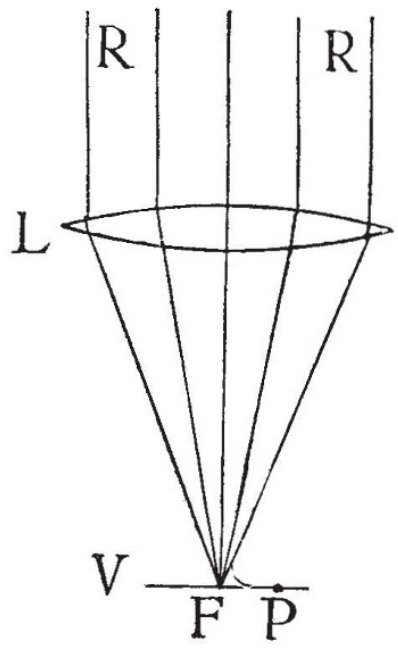

FIG. 2.

the instrument, one being reflected by the mirrors I and 2 , the other by 4 and 3 . The fact that in these circumstances interference fringes appear in the field of view proves that a quantum must reach from one of the outer mirrors to the other. The distance between these mirrors was no less than 6 metres.

The discrepancy between these estimates of the size of a quantum, according to which it would be too big to enter our eye, and, on the other hand, the notion that it is small enough to be captured by a single electron, is certainly very wide. Yet the laws of the two classes of phenomena about which we have reasoned, the phenomena of interference and those of photo-electricity, are so well established that there can be no real contradiction between what we deduce from one class and from the other; it must after all be possible to reconcile the different ideas. Here is an important problem for the physics of the immediate future. We cannot help thinking that the solution will be found in some happy combination of extended waves and concentrated quanta, the waves being made responsible for interference and the quanta for photoelectricity.

$$
\text { NO. } 2843 \text {, VOL. I I } 3]
$$

